\title{
Killing-Yano Cotton currents
}

\author{
Ulf Lindström ${ }^{a, b}$ and Özgür Sarığlu ${ }^{a}$ \\ ${ }^{a}$ Department of Physics, Faculty of Arts and Sciences, Middle East Technical University, \\ 06800, Ankara, Turkey \\ ${ }^{b}$ Department of Physics and Astronomy, Theoretical Physics, Uppsala University, \\ SE-751 20, Uppsala, Sweden \\ E-mail: ulf.lindstrom@physics.uu.se, sarioglu@metu.edu.tr
}

ABSTRACT: We discuss conserved currents constructed from the Cotton tensor and (conformal) Killing-Yano tensors (KYTs). We consider the corresponding charges generally and then exemplify with the four-dimensional Plebański-Demiański metric where they are proportional to the sum of the squares of the electric and the magnetic charges. As part of the derivation, we also find the two conformal Killing-Yano tensors of the PlebańskiDemiański metric in the recently introduced coordinates of Podolsky and Vratny. The construction of asymptotic charges for the Cotton current is elucidated and compared to the three-dimensional construction in Topologically Massive Gravity. For the threedimensional case, we also give a conformal superspace multiplet that contains the Cotton current in the bosonic sector. In a mathematical section, we derive potentials for the currents, find identities for conformal KYTs and for KYTs in torsionful backgrounds.

Keywords: Classical Theories of Gravity, Differential and Algebraic Geometry, Supergravity Models

ArXiv EPrint: 2110.03470 


\section{Contents}

1 Introduction 1

2 The Cotton current 2

3 Cotton charges for the Plebański-Demiański metric 4

3.1 An "improved" version 6

$\begin{array}{lll}4 & 3 D & \text { results }\end{array}$

4.1 The super Cotton current in $3 D$ conformal supergravity 8

$\begin{array}{lll}\text { 4.1.1 } 3 D \text { conformal superspace supergravity } & 8\end{array}$

$\begin{array}{ll}\text { 4.1.2 The super Cotton current } & 9\end{array}$

$\begin{array}{ll}4.2 \text { Currents from linearization? } & 10\end{array}$

5 Potentials \& new mathematical relations $\quad 12$

$\begin{array}{lll}5.1 \text { A potential for } j^{a} & 12\end{array}$

$\begin{array}{ll}5.2 \text { A potential for } J^{a} & 12\end{array}$

5.3 Potentials and charges for the Kerr-Newman metric 14

$\begin{array}{lll}5.4 & \text { KYT and torsion } & 15\end{array}$

6 Conclusions 16

$\begin{array}{lr}\text { A Spinor algebra } & 16\end{array}$

\section{Introduction}

Killing-Yano tensors (KYTs) have long been studied. They play a role as square roots of second rank Killing tensors (KTs). In gravity, supergravity and string theory they are used to find hidden symmetries for particles and backgrounds, to separate variables in Hamilton-Jacobi equations and to study the symmetries of the Dirac equation and its super extensions.

General background on KTs and KYTs and their applications can be found in [1,2] and [3]. Some interesting aspects are: finding new supersymmetries, covered in "Susy in the sky" [4] and their role in string theory covered, e.g. in [5]. There are applications in General Relativity (GR) [6, 7] to $G$-structures [8, 9], to WZW models [10], to classical mechanics [11] and to symmetries of the Dirac operator and super Laplacians [12, 13]. Supersymmetric conformal KTs and KYTs are discussed in [14], in [15] and [16]. Finally KTs arise in the context of hyperKähler geometry [17].

The present paper grew out of the results in [18] which, in turn, was inspired by a result of Kastor and Traschen $[19,20]$. These authors found a conserved current for an arbitrary 
rank KYT and it was used in certain backgrounds to construct asymptotic charges [21]. Here we continue and extend the discussion of conserved currents from [18]. In particular, we focus on a current constructed from the Cotton tensor and (conformal) KYTs. For this current we display the conserved charges in general, and for the four-dimensional $(4 D)$ case of the Plebański-Demiański metric in particular. In the process we derive the two conformal KYTs (CKYTs) needed in the new Podolsky-Vratny coordinates. We further discuss the special case of three dimensions $(3 D)$ at some length, finding obstructions to asymptotic charges, comparing to results in Topologically Massive Gravity (TMG) and generalizing our current to $3 D$ conformal supergravity in superspace. Continuing our mathematical study of KYTs, we finally include a section with identities for CKYTs.

The paper is organized as follows: in section 2 we define the Cotton current for an arbitrary dimension $D \geq 3$ and in section 3 we study it in $4 D$ using the Plebański-Demiański metric as an example. In section 4 we descend to $3 D$ and initiate a program for studying super KY currents in supergravity by generalizing the Cotton current to a supermultiplet in $3 D$ conformal supergravity. Here we also discuss linearization around a background carrying a second rank KYT and compare some results to the ones derived in [22]. Section 5 presents the potentials for the Cotton currents. The derivation of these involve some new mathematical relations for KYTs and CKYTs. To these we add a useful relation for the case of backgrounds with totally skew torsion. Section 6 contains our conclusions. A short appendix has the conventions for our spinor algebra.

The setting of our discussions is GR on a manifold $\mathcal{M}$ of dimension $D$ that carries a metric $g$ and a rank $n$ KYT $f$, or CKYT $k$. The coordinate indices are denoted by latin letters $a, b, \ldots$. When we consider extension to $3 D$ supergravity in section 4.1 , we use spinor indices and denote vector indices by pairs of these.

\section{The Cotton current}

In this section we introduce conserved currents constructed from the Cotton tensor and (C)KYTs. The Cotton tensor is defined in $D \geq 3$ dimensions as

$$
C_{a b c} \equiv 2(D-2) \nabla_{[c} S_{b] a}=2 \nabla_{[c} R_{b] a}-\frac{1}{(D-1)} g_{a[b} \nabla_{c]} R,
$$

where $R_{a b}$ is the Ricci tensor, $R$ is the curvature scalar and $S_{a b}$ is the Schouten tensor. In $D>3$ dimensions, it is related to the Weyl tensor $W_{a b c d}$ by

$$
C_{b d c}=\frac{D-2}{D-3} \nabla_{a} W_{b c d}^{a} .
$$

It is traceless on all index pairs and satisfies

$$
\begin{aligned}
C_{a b c} & =C_{a[b c]}, \\
C_{[a b c]} & =0, \\
\nabla^{a} C_{a b c} & =0 .
\end{aligned}
$$


We shall be interested in combining the Cotton tensor with a (C)KYT to construct conserved currents. A KYT of rank $n$ is an $n$-form $f$ that satisfies

$$
\nabla_{a} f_{b_{1} \ldots b_{n}}=\nabla_{[a} f_{\left.b_{1} \ldots b_{n}\right]}
$$

or equivalently

$$
\nabla_{(a} f_{\left.b_{1}\right) \ldots b_{n}}=0 \text {. }
$$

Similarly, a CKYT of rank $n$ is an $n$-form $k$ that satisfies

$$
\nabla_{a} k_{b_{1} \ldots b_{n}}=\nabla_{[a} k_{\left.b_{1} \ldots b_{n}\right]}+\frac{n}{D-n+1} g_{a\left[b_{1}\right.} \bar{k}_{\left.b_{2} \ldots b_{n}\right]},
$$

where

$$
\bar{k}_{b_{1} \ldots b_{n-1}} \equiv \nabla_{c} k_{b_{1} \ldots b_{n-1}}^{c} .
$$

Consider the following combinations of the Cotton tensor $C$, a rank-2 KYT $f$ and a rank-2 CKYT $k$ :

$$
j^{a} \equiv C^{a b c} f_{b c} \quad \text { and } \quad J^{a} \equiv C^{a b c} k_{b c}
$$

Note that

$$
\nabla_{a} j^{a}=\left(\nabla_{a} C^{a b c}\right) f_{b c}+C^{a b c} \nabla_{a} f_{b c}=0+C^{a b c} \nabla_{[a} f_{b c]}=0
$$

where the last two relations in (2.3) have been invoked. Hence $j^{a}$ is covariantly conserved. Similarly, we find that $J^{a}$ is also covariantly conserved: there is an additional term in the calculation corresponding to $(2.9)$

$$
\nabla_{a} J^{a}=0+\frac{2}{D-1} C^{a b c} g_{a[b} \bar{k}_{c]}=0,
$$

where the last equality follows from the fact that the Cotton tensor is traceless.

Since both the Cotton tensor and the CKYT are related to conformal properties of the manifold, one may ask about the transformation properties of $J^{a}$ under Weyl rescalings of the metric $g_{a b}^{\prime}=e^{C} g_{a b}$. The Cotton tensor transforms as

$$
C_{a b c}^{\prime}=C_{a b c}-\frac{1}{2}(D-2)\left(\partial_{d} C\right) W_{a b c}^{d} .
$$

A second rank CKYT transforms as [2]

$$
k_{a b}^{\prime}=e^{3 C / 2} k_{a b} \quad \text { and } \quad \bar{k}_{c}^{\prime}=e^{C / 2}\left(\bar{k}_{c}+\frac{1}{2}\left(\partial_{a} C\right) k_{c}^{a}\right) .
$$

From (2.11) and (2.12) it follows that 1-form current $J_{a}=C_{a b c} k^{b c}$ transforms as

$$
J_{a}^{\prime} \equiv C_{a b c}^{\prime} k^{\prime b c}=e^{-C / 2}\left(C_{a b c}-\frac{1}{2}(D-2)\left(\partial_{d} C\right) W_{a b c}^{d}\right) k^{b c},
$$

and the vector current $J^{a}$ as

$$
J^{\prime a}=e^{-3 C / 2}\left(C^{a}{ }_{b c}-\frac{1}{2}(D-2)\left(\partial_{d} C\right) W_{b c}^{d a}\right) k^{b c} .
$$


This is clearly not a conformal scaling in general. In $3 D$ the Weyl tensor does not exist which means that the covariant current scales with $e^{-C / 2}$ and that the contravariant current scales with $e^{-3 C / 2}$. This holds in arbitrary $D$ for conformally flat metrics or for constant scalings $C$.

We emphasize that the Cotton current (2.8) defined here is unrelated to the other currents discussed in [18]. In particular, the currents in $[18,19]$ are at most second order in the derivatives of the metric while the Cotton current (2.8) is of third order. The current in [19] is defined for arbitrary rank $n$ KYT and reduces to the Einstein current for $n=1$, while our Cotton current (2.8) is only defined for rank 2 . Moreover, we examine the Cotton current for $n=2$ in $3 D$, its relation to the discussion in [22] and its generalization to supergravity in section 4 . The current in [19] on the other hand vanishes for $n=2$ in $3 D$, since it is proportional to the Weyl tensor as shown in [18].

\section{Cotton charges for the Plebański-Demiański metric}

In this section we want to give a nontrivial example where the newly introduced Cotton current $J^{a}$ (2.8) can be used for defining a conserved charge. For that purpose we resort to the well-known, non-vacuum solution of GR, the celebrated Plebański-Demiański metric $[23,24]$

$$
d s^{2}=\Omega^{2}\left(-\frac{Q(r)\left(d \tau-p^{2} d \sigma\right)^{2}}{r^{2}+p^{2}}+\frac{P(p)\left(d \tau+r^{2} d \sigma\right)^{2}}{r^{2}+p^{2}}+\frac{r^{2}+p^{2}}{P(p)} d p^{2}+\frac{r^{2}+p^{2}}{Q(r)} d r^{2}\right),
$$

which solves the cosmological Einstein-Maxwell equations $G_{a b}=2 T_{a b}-\Lambda g_{a b}$ in $D=4$ when the metric functions $Q(r)$ and $P(p)$ are chosen as

$$
\begin{aligned}
& Q(r)=k+e^{2}+g^{2}-2 m r+\epsilon r^{2}-2 n r^{3}-(k+\Lambda / 3) r^{4}, \\
& P(p)=k+2 n p-\epsilon p^{2}+2 m p^{3}-\left(k+e^{2}+g^{2}+\Lambda / 3\right) p^{4},
\end{aligned}
$$

the conformal factor is

$$
\Omega(p, r)=(1-p r)^{-1}
$$

and the vector potential is

$$
A_{a} d x^{a}=-\frac{1}{r^{2}+p^{2}}\left(e r\left(d \tau-p^{2} d \sigma\right)+g p\left(d \tau+r^{2} d \sigma\right)\right) .
$$

Out of the seven $(m, n, e, g, \epsilon, k$ and $\Lambda)$ arbitrary real parameters, $e$ and $g$ represent the electric and magnetic charges, respectively, whereas $\Lambda$ is the cosmological constant [25]. As shown in [24], the Plebański-Demiański metric (3.1) (without using the special choices (3.2), (3.3) and (3.4)) has a rank-2 CKYT (5.24), which reads

$$
k=\Omega^{3}\left(p d r \wedge\left(d \tau-p^{2} d \sigma\right)+r d p \wedge\left(d \tau+r^{2} d \sigma\right)\right) .
$$

It turns out that its dual $h \equiv * k$ is also a rank-2 CKYT (5.24) given by [24]

$$
h=\Omega^{3}\left(r d r \wedge\left(p^{2} d \sigma-d \tau\right)+p d p \wedge\left(r^{2} d \sigma+d \tau\right)\right) .
$$


These ingredients can be used for calculating the Cotton tensor $C$ and the conformal Cotton currents $J_{k}$ (using (3.5)) and $J_{h}$ (using (3.6)). For the special choices (3.2), (3.3) and (3.4), we find that Cotton current vectors are

$$
J_{k}=\frac{4\left(e^{2}+g^{2}\right)(1-p r)^{4}}{\left(p^{2}+r^{2}\right)^{3}}\left(2 p^{2} r^{2} \partial_{\tau}+\left(r^{2}-p^{2}\right) \partial_{\sigma}\right)
$$

and

$$
J_{h}=\frac{4\left(e^{2}+g^{2}\right)(1-p r)^{4}}{\left(p^{2}+r^{2}\right)^{3}}\left(\left(p^{2}-r^{2}\right) \partial_{\tau}+2 \partial_{\sigma}\right) .
$$

It seems natural to interpret the coordinate $\tau$ as the global time function so that $t^{a}=\left(\partial_{\tau}\right)^{a}$ is a vector field on the spacetime satisfying $t^{a} \nabla_{a} \tau=1$. We may then foliate the spacetime (3.1) by Cauchy surfaces $\Sigma_{\tau}$ parametrized by $\tau$ such that the metric $g_{a b}$ (3.1) induces a spatial metric $\gamma_{a b}$ on each $\Sigma_{\tau}$ as

$$
\gamma_{a b}=g_{a b}+n_{a} n_{b}
$$

where $n^{a}$ is the unit normal vector field to the hypersurfaces $\Sigma_{\tau}$ :

$$
\begin{aligned}
n^{a} & =-\frac{1}{\Omega} \sqrt{\frac{p^{2}+r^{2}}{Q(r)-P(p)}}\left(\partial_{\tau}\right)^{a}, \\
n_{a} d x^{a} & =\frac{\Omega}{\sqrt{p^{2}+r^{2}}}\left(\sqrt{Q(r)-P(p)} d \tau-\frac{r^{2} P(p)+p^{2} Q(r)}{\sqrt{Q(r)-P(p)}} d \sigma\right),
\end{aligned}
$$

with $n^{a} n_{a}=-1$. The relevant piece of the volume element on the hypersurface $\Sigma_{\tau}$ is

$$
\sqrt{\gamma}=\frac{\left(p^{2}+r^{2}\right)^{3 / 2} \Omega^{3}}{\sqrt{Q(r)-P(p)}}, \quad \text { with } \quad \gamma \equiv \operatorname{det} \gamma_{a b} .
$$

These can be used in defining a conserved charge $\mathcal{Q}$ in the usual way as

$$
\mathcal{Q} \equiv \int_{\Sigma_{\tau}} d^{3} x \sqrt{\gamma} J^{a} n_{a}
$$

provided the metric functions satisfy $P(p)>0$ and $Q(r)>0$, as suggested by the form of (3.1), and $\tau$ can be thought of as the global time function as discussed. Using (3.7) and (3.8), we find explicitly that

$$
\begin{aligned}
& \mathcal{Q}_{k}=4\left(e^{2}+g^{2}\right) \int d r d p d \sigma \frac{r^{2} P(p)-p^{2} Q(r)}{\left(p^{2}+r^{2}\right)(P(p)-Q(r))}, \\
& \mathcal{Q}_{h}=4\left(e^{2}+g^{2}\right) \int d r d p d \sigma \frac{P(p)+Q(r)}{\left(p^{2}+r^{2}\right)(P(p)-Q(r))} .
\end{aligned}
$$

At this stage, it is worth emphasizing that our only aim is to give a "proof of principle", i.e. that the Cotton currents $J_{k}$ and $J_{h}$ can be used to come up with conserved charges $\mathcal{Q}_{k}$ and $\mathcal{Q}_{h}$ á la (3.9). So rather than going into the discussion of different cases ${ }^{1}$ that arise from studying the roots of the quartic functions $P(p)$ and $Q(r)$ to determine the ranges of the coordinates $p$ and $r$ (that guarantee that $P(p)>0$ and $Q(r)>0$ ), we assume that the coordinate patch(es) have properly chosen ranges for the variables $p, r$ and $\sigma$ such that the relevant 3-dimensional integrals in $\mathcal{Q}_{k}$ and $\mathcal{Q}_{h}$ (3.9) are convergent. Note that our Cotton charges are proportional to the sums of the squares of the physical electric and magnetic charges.

\footnotetext{
${ }^{1}$ See e.g. the invaluable work [25] for that.
} 


\subsection{An "improved" version}

In a recent work [26], the Plebański-Demiański metric (for the version with a vanishing cosmological constant) has been cast in a new "improved" form where the metric functions $P$ and $Q$ have been factorized, and the parameters have been redefined to make their physical meaning more accessible (See the beautiful work [26] for details). Here we want to examine how this new version plays along with the Cotton current we propose.

The new kid on the block reads [26]

$$
\begin{aligned}
d s^{2}=\frac{1}{\Omega^{2}} & \left(-\frac{Q}{\rho^{2}}\left[d t-\left(a \sin ^{2} \theta+4 \ell \sin ^{2}(\theta / 2)\right) d \varphi\right]^{2}+\frac{\rho^{2}}{Q} d r^{2}\right. \\
& \left.+\frac{\rho^{2}}{P} d \theta^{2}+\frac{P}{\rho^{2}} \sin ^{2} \theta\left[a d t-\left(r^{2}+(a+\ell)^{2}\right) d \varphi\right]^{2}\right),
\end{aligned}
$$

where

$$
\begin{aligned}
\Omega & =1-\frac{\alpha a}{a^{2}+\ell^{2}} r(\ell+a \cos \theta), \\
\rho^{2} & =r^{2}+(\ell+a \cos \theta)^{2}, \\
P(\theta) & =\left(1-\frac{\alpha a}{a^{2}+\ell^{2}} r_{+}(\ell+a \cos \theta)\right)\left(1-\frac{\alpha a}{a^{2}+\ell^{2}} r_{-}(\ell+a \cos \theta)\right), \\
Q(r) & =\left(r-r_{+}\right)\left(r-r_{-}\right)\left(1+\alpha a \frac{a-\ell}{a^{2}+\ell^{2}} r\right)\left(1-\alpha a \frac{a+\ell}{a^{2}+\ell^{2}} r\right),
\end{aligned}
$$

and the special roots of $Q(r)$, that determine the two black-hole horizons are located at

$$
r_{ \pm} \equiv m \pm \sqrt{m^{2}+\ell^{2}-a^{2}-e^{2}-g^{2}} .
$$

There are now six (non-negative) real parameters: $e$ and $g$ are still the electric and magnetic charges, respectively, $m$ is the mass, $a$ is the "Kerr-like rotation", $\ell$ is the NUT parameter and $\alpha$ is acceleration [26]. This metric solves the Einstein-Maxwell equations $G_{a b}=2 T_{a b}$ with the vector potential

$$
A_{a} d x^{a}=-\sqrt{e^{2}+g^{2}} \frac{r}{\rho^{2}}\left[d t-\left(a \sin ^{2} \theta+4 \ell \sin ^{2}(\theta / 2)\right) d \varphi\right] .
$$

The two rank-2 CKYTs of (3.12) are ${ }^{2}$

$$
\begin{aligned}
\tilde{k}=\frac{1}{\Omega^{3}} & \left(-r \sin \theta d \theta \wedge\left[a d t-\left(r^{2}+(a+\ell)^{2}\right) d \varphi\right]\right. \\
& \left.+(\ell+a \cos \theta) d r \wedge\left[d t-\left(a \sin ^{2} \theta+4 \ell \sin ^{2}(\theta / 2)\right) d \varphi\right]\right), \\
\tilde{h}=\frac{1}{\Omega^{3}}( & r d r \wedge\left[d t-\left(a \sin ^{2} \theta+4 \ell \sin ^{2}(\theta / 2)\right) d \varphi\right] \\
& \left.+(\ell+a \cos \theta) \sin \theta d \theta \wedge\left[a d t-\left(r^{2}+(a+\ell)^{2}\right) d \varphi\right]\right) .
\end{aligned}
$$

\footnotetext{
${ }^{2}$ To our knowledge, these are new but presumably they can be obtained from the ones in [24] by employing judicious coordinate transformations.
} 
Unfortunately though, the analogous calculations for determining the charge $\mathcal{Q}$ (3.9) for the most general form of (3.12) with its CKYTs (3.14) and (3.15) turn out to be too complicated. Even though we have explicitly determined the analogous integrands as in (3.10) and (3.11), they are best left undisplayed. To have a more manageable example, we set the "Kerr-like rotation parameter" $a=0$ in the metric (3.12), which also makes $\Omega=1$ and $P=1[26]$ :

$$
d s^{2}=-\frac{Q}{\rho^{2}}\left(d t-4 \ell \sin ^{2}(\theta / 2) d \varphi\right)^{2}+\frac{\rho^{2}}{Q} d r^{2}+\left(r^{2}+\ell^{2}\right)\left(d \theta^{2}+\sin ^{2} \theta d \varphi^{2}\right),
$$

where we now have

$$
\begin{aligned}
Q(r) & =\left(r-r_{+}\right)\left(r-r_{-}\right), \quad \text { with } \quad r_{ \pm} \equiv m \pm \sqrt{m^{2}+\ell^{2}-e^{2}-g^{2}}, \\
\rho^{2} & =r^{2}+\ell^{2}
\end{aligned}
$$

The vector potential becomes

$$
A_{a} d x^{a}=-\sqrt{e^{2}+g^{2}} \frac{r}{\rho^{2}}\left(d t-4 \ell \sin ^{2}(\theta / 2) d \varphi\right),
$$

and (3.16) describes the non-singular charged Taub-NUT spacetime, which is also asymptotically flat as $r \rightarrow \pm \infty$ away from the $\theta=\pi$ axis [26].

It turns out that setting $a=0$ in $\tilde{k}(3.14)$,

$$
\hat{k}=r\left(r^{2}+\ell^{2}\right) \sin \theta d \theta \wedge d \varphi+\ell d r \wedge d t-4 \ell^{2} \sin ^{2}(\theta / 2) d r \wedge d \varphi,
$$

makes $\hat{k}$ (3.17) a KYT, not a CKYT, of (3.16). So we can not use it to define a Cotton current $J^{a}$, as was done previously, but it can be used to define the Cotton current $j^{a}(2.8)$. However, the $a=0$ version of $\tilde{h}$ (3.15) is still a CKYT of (3.16):

$$
\hat{h}=r d r \wedge d t-4 \ell r \sin ^{2}(\theta / 2) d r \wedge d \varphi-\ell\left(r^{2}+\ell^{2}\right) \sin \theta d \theta \wedge d \varphi .
$$

With these ingredients, we have the Cotton current vectors

$$
\begin{aligned}
j_{\hat{k}} & =0, \quad \text { so that we trivially have } \mathcal{Q}_{\hat{k}}=0, \\
J_{\hat{h}} & =\frac{8\left(e^{2}+g^{2}\right)\left(r-r_{+}\right)\left(r-r_{-}\right)}{\left(r^{2}+\ell^{2}\right)^{3}}\left(-\partial_{t}+4 \ell \sin ^{2}(\theta / 2) \partial_{\varphi}\right) .
\end{aligned}
$$

Finally, we find the charge $\mathcal{Q}_{\hat{h}}$ to be ${ }^{3}$

$$
\mathcal{Q}_{\hat{h}}=32 \pi\left(e^{2}+g^{2}\right) \int_{-\infty}^{\infty} \frac{d r}{r^{2}+\ell^{2}}=\frac{32 \pi^{2}}{\ell}\left(e^{2}+g^{2}\right) .
$$

\section{$43 D$ results}

The Cotton tensor is particularly relevant in $3 D$ where it plays a role similar to that of the Weyl tensor in higher dimensions. In this section we discuss various properties of the Cotton current pertaining to $3 D$.

\footnotetext{
${ }^{3}$ Here the ranges of the metric functions are $r \in(-\infty, \infty), \theta \in[0, \pi]$ and $\varphi \in[0,2 \pi][26]$.
} 


\subsection{The super Cotton current in $3 D$ conformal supergravity}

In this subsection we introduce the conformal supergravity extension of the Cotton current.

\subsection{1 $3 D$ conformal superspace supergravity}

In $3 D, \mathcal{N}=1$ Conformal Superspace Conformal Supergravity is defined by the following $\operatorname{algebra}^{4}[27]$

$$
\begin{aligned}
\left\{\nabla_{\alpha}, \nabla_{\beta}\right\} & =2 i \nabla_{\alpha \beta}, \\
{\left[\nabla_{a}, \nabla_{\alpha}\right] } & =\frac{1}{4}\left(\gamma_{a}\right)_{\alpha}{ }_{\alpha} W_{\beta \gamma \delta} K^{\gamma \delta} \\
{\left[\nabla_{a}, \nabla_{b}\right] } & =-\frac{i}{8} \epsilon_{a b c}\left(\gamma^{c}\right)^{\alpha \beta} \nabla_{\alpha} W_{\beta \gamma \delta} K^{\gamma \delta}-\frac{1}{4} \epsilon_{a b c}\left(\gamma^{c}\right)^{\alpha \beta} W_{\alpha \beta \gamma} S^{\gamma},
\end{aligned}
$$

where $K$ is the generator of special conformal transformation and $S$ is the generator of $S$ supersymmetry. The whole super conformal group has been gauged and dilation curvature has been set to zero. The Lorentz generator $M$ and the translation generator $P$ give rise to the covariant derivatives. We shall need the properties of the super Cotton tensor $W$ [28]

$$
\begin{aligned}
W_{\alpha \beta \gamma} & =W_{(\alpha \beta \gamma)}, \\
\nabla^{\alpha} W_{\alpha \beta \gamma} & =0, \\
K_{a} W_{\alpha \beta \gamma} & =0,
\end{aligned}
$$

where the last relation says that $W$ is a primary superfield.

From

$$
\nabla_{\alpha} \nabla_{\beta}=i \nabla_{\alpha \beta}+\frac{1}{2} \epsilon_{\alpha \beta} \nabla^{\gamma} \nabla_{\gamma}
$$

and the fact that, on a primary field $\left[\nabla_{\alpha \beta}, \nabla_{\gamma}\right]=0$, it follows that

$$
\begin{aligned}
\nabla^{2} \nabla_{\alpha} & =-\nabla_{\alpha} \nabla^{2}=2 i \nabla^{\gamma} \nabla_{\gamma \alpha}, \\
\left\{\nabla^{2}, \nabla_{\alpha}\right\} & =0, \quad\left[\nabla^{2}, \nabla_{\alpha}\right]=4 i \nabla^{\gamma} \nabla_{\gamma \alpha}, \\
\nabla^{\gamma} \nabla_{\alpha} \nabla_{\gamma} & =0 .
\end{aligned}
$$

We take a superconformal Killing supervector field $\xi$ to be given by

$$
\xi=\xi^{a} \nabla_{a}+\xi^{\alpha} \nabla_{\alpha}
$$

where $\xi^{a}$ is a primary and where the components are related by

$$
\nabla_{\alpha} \xi_{\mu \nu}=4 i \epsilon_{\alpha(\mu} \xi_{\nu)}
$$

It follows that

$$
\begin{aligned}
\nabla^{\alpha} \xi^{\mu \nu} & =-4 i \epsilon^{\alpha(\mu} \xi^{\nu)}, \\
\nabla_{(\alpha} \xi_{\mu \nu)} & =0
\end{aligned}
$$

\footnotetext{
${ }^{4}$ See appendix A for conventions.
} 


$$
\begin{aligned}
\nabla_{(\alpha} \xi_{\beta)} & =\frac{1}{4} \nabla_{(\alpha}^{\gamma} \xi_{\beta) \gamma}, \\
\nabla_{\gamma} \xi^{\gamma} & =\frac{1}{3} \nabla_{a} \xi^{a}, \\
\xi^{\alpha} & =\frac{i}{6}\left(\gamma_{a}\right)^{\alpha \beta} \nabla_{\beta} \xi^{a}, \\
\nabla^{2} \xi_{\alpha} & =i \frac{2}{3} \nabla_{\alpha}^{\gamma} \xi_{\gamma}, \\
\nabla_{(a} \xi_{b)} & =\frac{1}{3} \eta_{a b} \nabla_{c} \xi^{c}, \\
\nabla^{\beta \gamma} \xi^{\alpha} & =-\frac{2}{3} \epsilon^{\alpha(\beta} \nabla_{\sigma}^{\gamma)} \xi^{\sigma},
\end{aligned}
$$

where the next to last relation defines a conformal Killing vector and the last one a conformal Killing spinor.

\subsubsection{The super Cotton current}

From the last line in (4.1) we see that

$$
C_{a b}=\nabla_{\alpha} W_{\beta \gamma \delta} \quad \text { and } \quad W_{\beta \gamma \delta}
$$

will correspond to the Cotton and the Cottino tensors at the component level [27]. Indeed $C_{a b}$ has the properties of the Cotton-York tensor

$$
\nabla_{a} C_{b}^{a}=0, \quad C_{a b}=C_{(a b)}, \quad C_{a}^{a}=0 .
$$

We may now construct a supergravity version of our Cotton current $J$ in (2.8). To this end we define

$$
\begin{aligned}
k_{\alpha} & =W_{\alpha \beta \gamma} \xi^{\beta \gamma} \\
k_{\alpha \beta} & =\nabla_{\alpha} k_{\beta}=\left(\nabla_{(\alpha} W_{\beta) \gamma \delta}\right) \xi^{\gamma \delta}+4 i W_{\alpha \beta \gamma} \xi^{\gamma} .
\end{aligned}
$$

These satisfy

$$
\begin{aligned}
\nabla^{\alpha} k_{\alpha} & =0, \\
\nabla^{\alpha \beta} k_{\alpha \beta} & =0,
\end{aligned}
$$

and the lowest component of the first part of $k_{\alpha \beta}$ is the bosonic Cotton current $J$ in (2.8).

In a more covariant form we have

$$
\left(k^{A}\right)=\left(k^{\alpha \beta}, k^{\alpha}\right)
$$

with

$$
\nabla_{A} k^{A}=0
$$




\subsection{Currents from linearization?}

Here we want to examine whether we can extend the discussion in subsection 6.1 of [18] to the currents introduced in section 2. So we linearize the metric around a background metric and assume that there exists a KYT asymptotically. If the linearized current defined with this KYT is still conserved, we may try to construct its asymptotic charges. For simplicity, consider only rank-2 KYTs in what follows. For brevity, let us start by reproducing some formulas that will be relevant for the ensuing discussion:

$$
\begin{aligned}
\left(\Gamma_{a b}^{c}\right)_{L} & =\frac{1}{2} \bar{g}^{c e}\left(\bar{\nabla}_{a} h_{b e}+\bar{\nabla}_{b} h_{a e}-\bar{\nabla}_{e} h_{a b}\right), \\
\left(R_{b}^{a}\right)_{L} & =\frac{1}{2}\left(\bar{\nabla}^{c} \bar{\nabla}^{a} h_{b c}+\bar{\nabla}_{c} \bar{\nabla}_{b} h^{a c}-\bar{\nabla}^{a} \bar{\nabla}_{b} h-\bar{\square}_{b}^{a}\right)-h^{a c} \bar{R}_{b c}, \\
R_{L} & =\bar{\nabla}_{a} \bar{\nabla}_{b} h^{a b}-\bar{\square} h-h^{a b} \bar{R}_{a b} .
\end{aligned}
$$

The Cotton current $j^{a}(2.8)$, and its linearization, is most relevant in $D=3$. This is due to the special role played by the Cotton tensor in the field equations of TMG [29, 30], so let us set $D=3$ in what follows and consider the linearized current

$$
j_{L}^{a} \equiv 2\left(\nabla_{c} S^{a}{ }_{b}\right)_{L} \bar{f}^{b c} .
$$

Since

$$
\left(\nabla_{c} S_{b}^{a}\right)_{L}=\bar{\nabla}_{c}\left(S_{b}^{a}\right)_{L}+\left(\Gamma_{c d}^{a}\right)_{L} \bar{S}_{b}^{d}-\left(\Gamma_{c b}^{d}\right)_{L} \bar{S}_{d}^{a}
$$

and

$$
\bar{S}_{a b} \bar{f}^{a c}+\bar{S}^{a c} \bar{f}_{a b}=0
$$

that follows from $R_{a b} f^{a c}+R^{a c} f_{a b}=0$ [18], we find

$$
j_{L}^{a}=\bar{\nabla}_{c} \bar{\ell}^{a c}
$$

where $\bar{\ell}^{a c}=2 \bar{f}^{b c}\left(S^{a}{ }_{b}\right)_{L}$. We emphasize that this result has been obtained by working with a generic background. Unfortunately though, we do not have $\bar{\ell}^{a c}=\bar{\ell}^{[a c]}$, or that $\bar{\nabla}_{a} j_{L}^{a}=0$ even when the background is maximally symmetric. So we cannot use $j^{a}$ to define asymptotic $\mathrm{AD}$ charges.

It is worth comparing this result with the relevant piece of the linearized current employed in [22] to define conserved gravitational charges in TMG. There the part relevant to our discussion is defined through the linearization of the York tensor ${ }^{5} C^{a b}$, which is symmetric, traceless and identically conserved, and defined as

$$
C^{a b}=\epsilon^{a c d} \nabla_{c} S_{d}^{b}
$$

and is the dual of the Cotton tensor, i.e. $C^{b}{ }_{c d}=\epsilon_{a c d} C^{a b}$. In [22], it is shown that it is always possible to write

$$
C_{L}^{a b} \bar{\xi}_{b}=\bar{\nabla}_{b}\left(\overline{\mathcal{F}}^{[a b]}(\bar{\xi})\right),
$$

\footnotetext{
${ }^{5}$ We use the term "York tensor" to denote the dual of the Cotton tensor, which is also called the "Cotton tensor" or "Cotton-York tensor" in the literature.
} 
where $\bar{\xi}^{b}$ is a background Killing vector, $\bar{\Xi}^{a} \equiv \bar{\epsilon}^{a b c} \bar{\nabla}_{b} \bar{\xi}_{c}$ is another Killing vector constructed out of $\bar{\xi}$ and

$$
\begin{aligned}
\mathcal{F}^{a b}(\bar{\xi}) & =\frac{1}{2}\left(\mathcal{F}_{\mathrm{E}}^{a b}(\bar{\Xi})+\bar{\epsilon}^{c b d} \bar{\xi}_{c}\left(G_{d}{ }^{a}\right)_{L}+\bar{\epsilon}^{a b d} \bar{\xi}_{c}\left(G_{d}{ }^{c}\right)_{L}+\bar{\epsilon}^{a c d} \bar{\xi}_{c}\left(G_{d}{ }^{b}\right)_{L}\right), \\
\mathcal{F}_{\mathrm{E}}^{a b}(\bar{\Xi}) & =\bar{\Xi}_{c} \bar{\nabla}^{[a} h^{b] c}+\bar{\Xi}^{[b} \bar{\nabla}_{c} h^{a] c}+h^{c[b} \bar{\nabla}_{c} \bar{\Xi}^{a]}+\bar{\Xi}^{[a} \bar{\nabla}^{b]} h+\frac{1}{2} h \bar{\nabla}^{[a} \bar{\Xi}^{b]}, \\
\left(G^{a}{ }_{b}\right)_{L} & =\left(R^{a}{ }_{b}\right)_{L}-\frac{1}{2} \delta^{a}{ }_{b} R_{L}-2 \Lambda h^{a}{ }_{b}, \\
R_{a b}^{L} & =\frac{1}{2}\left(\bar{\nabla}^{c} \bar{\nabla}_{b} h_{a c}+\bar{\nabla}^{c} \bar{\nabla}_{a} h_{b c}-\bar{\square} h_{a b}-\bar{\nabla}_{a} \bar{\nabla}_{b} h\right), \\
R_{L} & =\bar{\nabla}^{a} \bar{\nabla}^{b} h_{a b}-\bar{\square} h-2 \Lambda h,
\end{aligned}
$$

where all contractions, raising and lowering of indices are performed with respect to the maximally symmetric background $\bar{g}_{a b}$ for which $\bar{R}_{a b}=2 \Lambda \bar{g}_{a b}$ and $\bar{R}=6 \Lambda$, with $\Lambda<0$, $h_{a b} \equiv g_{a b}-\bar{g}_{a b}$ denotes the deviations from the background, $h \equiv \bar{g}^{a b} h_{a b}, \bar{\nabla}$ indicates the covariant derivative with respect to the background and $\bar{\square} \equiv \bar{\nabla}^{a} \bar{\nabla}_{a}$.

Since we have $\left(\nabla_{c} S_{d}^{b}\right)_{L}=\bar{\nabla}_{c}\left(S_{d}^{b}\right)_{L}$ on such a background, it follows that $C_{L}^{a b}=$ $\bar{\epsilon}^{a c d} \bar{\nabla}_{c}\left(S_{d}^{b}\right)_{L}$. This leads to the conclusion that it is possible to write

$$
\bar{\epsilon}_{a c d} C_{L}^{a b} \bar{\xi}_{b}=\left(C_{c d}^{b}\right)_{L} \bar{\xi}_{b}=\bar{\nabla}_{b}\left(\bar{\epsilon}_{a c d} \overline{\mathcal{F}}^{[a b]}\right)
$$

In fact a close examination of (4.19) and (4.20), and the way the Killing vector $\bar{\Xi}$ has been defined above, reveals that all is indeed consistent with (4.18).

From another perspective, our proposed current (4.15) would have been satisfactory if we could write

$$
\left(C^{b}{ }_{c d}\right)_{L} \bar{f}^{c d}=\left(\bar{\epsilon}_{a c d} \bar{f}^{c d}\right) C_{L}^{a b}=: C_{L}^{a b} \bar{\zeta}_{a}
$$

where the would-be background Killing vector $\bar{\zeta}^{a}$, satisfying $\bar{\nabla}_{(a} \bar{\zeta}_{b)}=0$, would let us write

$$
\left(C^{b}{ }_{c d}\right)_{L} \bar{f}^{c d}=\bar{\nabla}_{a}\left(\overline{\mathcal{F}}^{[a b]}(\bar{\zeta})\right)
$$

on a par with (4.18). However, it is well known that $\bar{\zeta}_{a} \equiv \bar{\epsilon}_{a c d} \bar{f}^{c d}$ is a closed conformal Killing vector satisfying

$$
\bar{\nabla}_{(a} \bar{\zeta}_{b)}=\frac{1}{3}\left(\bar{\nabla}_{c} \bar{\zeta}^{c}\right) \bar{g}_{a b}
$$

Hence $j^{a}$ does not lead to asymptotic AD charges.

As a final note, we would like to point out to an interesting observation we made as we were trying out various ideas presented here using the renowned BTZ metric [31] considered as a solution to TMG:

$$
d s^{2}=\left(M-\frac{r^{2}}{\ell^{2}}\right) d t^{2}-J d t d \theta+r^{2} d \theta^{2}+\frac{d r^{2}}{-M+\frac{r^{2}}{\ell^{2}}+\frac{J^{2}}{4 r^{2}}} .
$$

The background to work with is locally AdS and obtained by setting $M=J=0$ in (4.21):

$$
d \bar{s}^{2}=-\frac{r^{2}}{\ell^{2}} d t^{2}+r^{2} d \theta^{2}+\frac{\ell^{2}}{r^{2}} d r^{2} .
$$


The details of how to determine the energy and angular momentum of (4.21) in TMG using the maximally symmetric background (4.22) can be found in [21]. Here we just want to point out to the remarkable result that the linearized York tensor $C_{L}^{a b}$ that goes into the current (4.18) identically vanishes in this setting, but is nevertheless able to produce sensible $\overline{\mathcal{F}}^{[a b]}(\bar{\xi})$ as explained in [21].

\section{Potentials \& new mathematical relations}

In this section we find the potentials for the Cotton currents $j^{a}$ and $J^{a}$, uncovering some interesting new relations for CKYTs en route. We collect these and a relation for KYTs in torsionful geometries, continuing the mathematical investigations in [18].

A covariantly conserved antisymmetric rank $n$ tensor represents a co-closed $n$-form. By the Poincaré lemma extended to the exterior co-derivative this means that it is equal to the co-derivative of an $(n+1)$-form in an appropriately chosen open set. We refer to this $(n+1)$-form as a potential for the conserved tensor. It should thus be possible to write our conserved currents $j^{a}$ and $J^{a}$ as covariant divergences of such potentials.

\subsection{A potential for $j^{a}$}

For $j^{a}$ this is straightforward using the relation

$$
f^{a b} \nabla_{a} G_{b c}=0
$$

recently proven in [18]. It implies that the current reduces to the desired form $j^{a}=\nabla_{c} \ell^{a c}$ :

$$
j^{a}=\left(\frac{D-2}{D-1}\right) f^{a c} \nabla_{c} R=\nabla_{c}\left(\left(\frac{D-2}{D-1}\right) f^{a c} R\right),
$$

or, equivalently,

$$
\ell^{a c}=\left(\frac{D-2}{D-1}\right) f^{a c} R
$$

\subsection{A potential for $J^{a}$}

To find the potential for the Cotton current $J^{a}$ involving a second rank CKYT, we need to develop more tools.

The identity [19]

$$
\nabla_{a} \nabla_{b} f_{c_{1} \ldots c_{n}}=(-1)^{n+1} \frac{(n+1)}{2} R_{a\left[b c_{1}\right.}^{d} f_{\left.c_{2} \ldots c_{n}\right] d},
$$

may be generalized to CKYTs (2.6) (as well as to geometries with torsion, see below). From (2.6), a second rank CKYT $k$ satisfies

$$
\nabla_{a} k_{b c}=\nabla_{[a} k_{b c]}+\frac{2}{D-1} g_{a[b} \bar{k}_{c]} .
$$

The modified identity corresponding to (5.4) then reads (see e.g. [32]):

$$
\nabla_{a} \nabla_{b} k_{c d}=-\frac{3}{2} R_{a[b c}^{e} k_{d] e}-\frac{3}{D-1} g_{a[b} \nabla_{c} \bar{k}_{d]}+\frac{2}{D-1} \nabla_{a}\left(g_{b[c} \bar{k}_{d]}\right) .
$$


We note in passing that for a closed CKYT, i.e., when the first term on the r.h.s. of (5.5) vanishes, the relation (5.6) implies no restriction on $\bar{k}$.

From (5.6) we derive the following identities

$$
\begin{aligned}
\nabla_{a} \bar{k}^{a} & =\nabla_{a} \nabla_{b} k^{b a}=0, \quad(D-2) \nabla_{(a} \bar{k}_{b)}=(D-1) R_{(a}^{c} k_{b) c}, \\
(D-4) \nabla_{[a} \bar{k}_{b]} & =(D-1)\left(R_{[a}^{c} k_{b] c}-\square k_{a b}+\frac{1}{2} k^{c d} R_{c d a b}\right) .
\end{aligned}
$$

Note that, when the spacetime $g_{a b}$ is an Einstein space, i.e. $R_{a b}=K g_{a b}$ for a constant $K$, (5.7) shows that $\bar{k}^{a}$ is a Killing vector. The relations (5.7) and (5.8) can be used for writing

$$
\begin{aligned}
(D-4) \nabla_{a} \bar{k}_{b} & =(D-1)\left(\frac{D-3}{D-2} R_{a}^{c} k_{b c}-\frac{1}{D-2} R_{b}^{c} k_{a c}-\square k_{a b}+\frac{1}{2} k^{c d} R_{c d a b}\right), \\
(D-4) \nabla_{c} \nabla_{a} k_{b}^{c} & =\frac{2 D-5}{D-2} R_{a}^{c} k_{b c}-\frac{D-1}{D-2} R_{b}^{c} k_{a c}+\frac{3}{2} k^{c d} R_{c d a b}-(D-1) \square k_{a b} .
\end{aligned}
$$

Furthermore, when $D=4$, the left hand sides of (5.8), (5.9) and (5.10) vanish and yield nontrivial constraints on $k_{a b}$. Additionally, for a flat 4-dimensional spacetime, $k$ has to be harmonic.

We can also perform a calculation analogous to that of section 3.2 in [18] for a rank-2 CKYT. Using (5.5) and (5.6) in $\left[\nabla_{a}, \nabla_{b}\right] \nabla_{c} k_{d e}$, we first find

$$
\left[\nabla_{a}, \nabla_{b}\right] \nabla_{[c} k_{d e]}=\frac{3}{2}\left(\left(\nabla^{i} R_{b a[c d}\right) k_{e] i}-R_{b[c d}^{i} \nabla_{|a|} k_{e] i}+R_{a[c d}^{i} \nabla_{|b|} k_{e] i}\right)+\frac{6}{D-1} g_{[c[a} \nabla_{b]} \nabla_{d} \bar{k}_{e]} .
$$

Contracting the index pairs $(a, e)$ and $(b, c)$ in $(5.11)$, we arrive at

$$
k^{b c} \nabla_{c} G_{a b}+\frac{1}{D-1}\left(R \bar{k}_{a}+(D-4) R_{a c} \bar{k}^{c}+2(D-2) \nabla^{c} \nabla_{[c} \bar{k}_{a]}\right)=0,
$$

where $G_{a b}$ is the Einstein tensor. This correctly reduces to (5.1), its analog for a rank-2 KYT when $\bar{k}$ pieces are set to zero. Moreover, with (5.12), the current $J^{a}$ (2.8) can be expressed in an alternative form as

$$
J^{a}=\frac{1}{D-1}\left((D-2) k^{a c} \nabla_{c} R-2 R \bar{k}^{a}-2(D-4) R^{a c} \bar{k}_{c}-4(D-2) \nabla_{c} \nabla^{[c} \bar{k}^{a]}\right),
$$

in analogy to (5.2). Using (5.13), the covariant conservation of $J^{a}$ can be verified easily thanks to the observations

$$
2 \nabla_{a} \nabla_{c} \nabla^{[c} \bar{k}^{a]}=2 \nabla_{[a} \nabla_{c]} \nabla^{c} \bar{k}^{a}=\left[\nabla_{a}, \nabla_{c}\right] \nabla^{c} \bar{k}^{a}=0
$$

where the last equality follows from the definition of the Riemann tensor, and

$$
R^{a c} \nabla_{a} \bar{k}_{c}=R^{a c} \nabla_{(a} \bar{k}_{c)}=0
$$

where the last equality follows using (5.7). 
Note that we may alternatively write $J^{a}$ as

$$
\begin{aligned}
J^{a} & =\nabla_{c}\left(\left(\frac{D-2}{D-1}\right)\left(R k^{a c}-4 \nabla^{[c} \bar{k}^{a]}\right)\right)-2\left(\frac{D-4}{D-1}\right) G^{a c} \bar{k}_{c}, \\
& =\nabla_{c}\left(\left(\frac{D-2}{D-1}\right) R k^{a c}+2 G^{a b} k_{b}^{c}\right)+2 G^{a c} \bar{k}_{c}+\left(\frac{D-2}{D-1}\right) R \bar{k}^{a} . \\
& =\nabla_{c}\left(\left(\frac{D-2}{D-1}\right) R k^{a c}+2 G^{b[a} k_{b}^{c]}\right)+2\left(\frac{D-2}{D-1}\right) G^{a c} \bar{k}_{c} .
\end{aligned}
$$

Here the first line follows from (5.13), whereas the second one from using (2.1) and (2.8). The current can be written as a total divergence as $J^{a}=\nabla_{c} \ell^{a c}$ by equating the first and the third lines, eliminating the $G^{a c} \bar{k}_{c}$ bits, where

$$
\ell^{a c}=\frac{2(D-4)}{D-3} G^{b[a} k_{b}^{c]}+\frac{2(D-2)^{2}}{(D-1)(D-3)} \nabla^{[a} \bar{k}^{c]}+\left(\frac{D-2}{D-1}\right) R k^{a c}
$$

Note that $k_{a b}$ becomes a KYT when $\bar{k}^{a}=0$. Agreement between (5.15) and (5.3) then follows by observing that

$$
\nabla_{c}\left(G^{b[a} f^{c]} b\right)=0
$$

for a KYT $f_{a b}[18]$. This also shows the consistency of the following useful relation unveiled in the derivation of $(5.15)$

$$
G^{a c} \bar{k}_{c}=\left(\frac{D-1}{D-3}\right) \nabla_{c}\left(G^{b[c} k_{b}^{a]}+\left(\frac{D-2}{D-1}\right) \nabla^{[a} \bar{k}^{c]}\right) .
$$

\subsection{Potentials and charges for the Kerr-Newman metric}

Armed with the potential (5.15) we now return to the question of charges. We illustrate how the potential simplifies in $4 D$ with vanishing curvature scalar $R=0$.

An example of this is the Kerr-Newman metric, obtained by setting the acceleration $\alpha=0$ and the NUT parameter $\ell=0$ in the metric (3.12), which again makes $\Omega=1$ and $P=1[26]$ :

$$
d s^{2}=-\frac{Q}{\rho^{2}}\left(d t-a \sin ^{2} \theta d \varphi\right)^{2}+\frac{\rho^{2}}{Q} d r^{2}+\rho^{2} d \theta^{2}+\frac{\sin ^{2} \theta}{\rho^{2}}\left(a d t-\left(r^{2}+a^{2}\right) d \varphi\right)^{2},
$$

where we now have

$$
\begin{aligned}
Q(r) & =\left(r-r_{+}\right)\left(r-r_{-}\right), \quad \text { with } \quad r_{ \pm} \equiv m \pm \sqrt{m^{2}-a^{2}-e^{2}-g^{2}} \\
\rho^{2} & =r^{2}+a^{2} \cos ^{2} \theta
\end{aligned}
$$

We again find a KYT by setting $\alpha=0$ and $\ell=0$ in $\tilde{k}$ (3.14) which again leads to $\mathcal{Q}_{\hat{k}}=0$ through $j=0$, which is also easy to see from the potential (5.2). The charge expression $\mathcal{Q}_{\hat{h}}$ is easier to calculate using the potential $\ell^{a c}$ (5.15), since now the first and the third terms trivially vanish, for the remaining CKYT

$$
\tilde{h}=r d r \wedge\left[d t-a \sin ^{2} \theta d \varphi\right]+a \cos \theta \sin \theta d \theta \wedge\left[a d t-\left(r^{2}+a^{2}\right) d \varphi\right] .
$$


We find

$$
\mathcal{Q}_{\tilde{h}}=16 \pi\left(e^{2}+g^{2}\right) \int_{r_{+}}^{\infty} d r \int_{0}^{\pi} d \theta \frac{\sin \theta\left(-a^{2} \cos ^{2} \theta+2 a^{2}+e^{2}+g^{2}-2 m r+r^{2}\right)}{\left(a^{2} \cos ^{2} \theta+r^{2}\right)\left(a^{2} \cos ^{2} \theta+e^{2}+g^{2}-2 m r+r^{2}\right)},
$$

which is certainly convergent and finite. The $\theta$ integral can be taken exactly:

$$
\begin{aligned}
\mathcal{Q}_{\tilde{h}}= & \frac{32 \pi}{a}\left(e^{2}+g^{2}\right) \\
& \times \int_{r_{+}}^{\infty} d r \frac{\left(\frac{\left(2 a^{2}+e^{2}+g^{2}+2 r(r-m)\right) \tan ^{-1}\left(\frac{a}{r}\right)}{r}-\frac{2\left(a^{2}+e^{2}+g^{2}-2 m r+r^{2}\right) \tan ^{-1}\left(\frac{a}{\sqrt{e^{2}+g^{2}-2 m r+r^{2}}}\right)}{\sqrt{e^{2}+g^{2}+r(r-2 m)}}\right)}{\left(e^{2}+g^{2}-2 m r\right)} .
\end{aligned}
$$

Unfortunately though we have not been able to evaluate the $r$ integral ${ }^{6}$. Alternatively, we can evaluate the $r$ integral first, but that leads to an equally challenging expression for the $\theta$ integral.

\subsection{KYT and torsion}

In section 4.1 the geometry is torsionful. This prompts the question of generalization of some of our formulae to such geometries. CKYTs with torsion have been discussed, e.g., in $[9,33]$ and [34]. Here we shall not be able to treat the general case, but limit ourselves to the special case of completely skew torsion. The torsionful covariant derivative is then

$$
\hat{\nabla}=\nabla^{(0)}+T
$$

where $\nabla_{a}^{(0)}$ is the Levi-Civita connection and $T$ represents the torsion two-form

$$
T^{a}=\frac{1}{2} T_{b c}^{a} e^{b} \wedge e^{c} .
$$

We consider a two-form $f$ which satisfies

$$
\hat{\nabla}_{(a} f_{b) c}=\nabla_{(a}^{(0)} f_{b) c}+T_{c(a}{ }^{d} f_{b) d}=0 .
$$

From this it follows that

$$
\hat{\nabla}_{a} f_{b c}=\hat{\nabla}_{[a} f_{b c]}, \quad \hat{\nabla}_{a} f_{c}^{a}=0,
$$

and we may take (5.22) or (5.23) as the definition of a KY two-form in the presence of torsion. For a general $n$ CKYT $k$ we equivalently define

$$
\hat{\nabla}_{a} k_{b_{1} \ldots b_{n}}=\hat{\nabla}_{[a} k_{\left.b_{1} \ldots b_{n}\right]}+\frac{n}{D-n+1} g_{a\left[b_{1}\right.} \bar{k}_{\left.b_{2} \ldots b_{n}\right]},
$$

where

$$
\bar{k}_{b_{1} \ldots b_{n-1}}:=\hat{\nabla}_{c} k_{b_{1} \ldots b_{n-1}}^{c} .
$$

For a KY two-form $f$, we then find, using the torsionful Bianchi identity

$$
\hat{R}_{[d b c]}^{e}=\nabla_{[d} T_{b c]}^{e}+T_{[d b}^{f} T_{c] f}^{e},
$$

that the identity corresponding to (5.4) becomes

$$
\hat{\nabla}_{a} \hat{\nabla}_{b} f_{c d}=\frac{3}{2} \hat{R}_{[b c|a|}^{e} f_{d] e}+\frac{3}{2}\left(\hat{\nabla}_{[d} T_{b c]}^{e}+T_{[d b}^{f} T_{c] f}^{e}\right) f_{e a}-4 \hat{R}_{[a b c}^{e} f_{d] e} .
$$

The explicit torsion expression for the last term follows from (5.26).

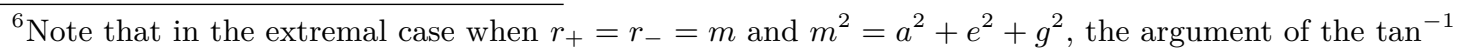
in the second piece at the lower limit $r=m$ becomes imaginary.
} 


\section{Conclusions}

In this paper we have introduced conserved currents based on the Cotton tensor and second rank (C)KYTs and derived their potentials. As an example we have studied the PlebańskiDemiański metric and found its two CKYTs in the new coordinates introduced in [26]. The corresponding Cotton charges are proportional to the sum of the squares of the electric and the magnetic charges in general. In the particular case of the charged Taub-NUT spacetime, which corresponds to setting the rotation parameter $a$ to zero, the charge for the non-vanishing CKYT is simply $32 \pi^{2}\left(e^{2}+g^{2}\right) / \ell$ with the NUT parameter $\ell$. Obviously, the conserved charge we find is a combination of fundamental charges already present in the metric. The existence of KYTs and conserved currents formed using them does not automatically lead to new charges. This is evident in the case of black holes where all the conserved charges are known by other means. However for other possibly exotic geometries, new and hitherto unearthed (hidden) charges could well arise. Therefore, there are other metrics waiting to be analyzed similarly, in particular in higher dimensions.

For the $3 D$ case we have discussed possible asymptotic charges, relations to results in TMG and constructed a generalization to conformal supergravity. This latter result opens up the possibility to construct similar currents for supergravities in higher dimensions.

The derivation of the potentials for the Cotton currents involve generalizations of identities for KYTs and CKYTs, and shows that there are still interesting geometric relations for these objects that deserve attention.

Note added. While this paper was a preprint, we were asked by D. Kubiznak what would happen if one considered the $\ell=0$ case, i.e., the Reissner-Nordström case, in the example leading to (3.20). The answer is that the whole discussion follows as before, but the range of the integral in (3.20) is from $r_{+}$to $\infty$ now, and one has a well-defined finite answer.

\section{Acknowledgments}

We thank Sergei Kuzenko for commenting on the supergravity part of the paper, and D. Kubiznak for comments. The research of U.L. is supported in part by the 2236 Co-Funded Scheme2 (CoCirculation2) of TÜBİTAK (Project No:120C067). ${ }^{7}$

\section{A Spinor algebra}

The $3 D$ spinor algebra we need is

$$
\begin{aligned}
\left\{\gamma_{a}, \gamma_{b}\right\} & =2 \eta_{a b}, \\
\epsilon^{\alpha \beta} \epsilon_{\beta \gamma} & =\delta_{\gamma}^{\alpha}, \quad \psi^{\alpha}=\epsilon^{\alpha \beta} \psi_{\beta}, \quad \psi_{\alpha}=\epsilon_{\alpha \beta} \psi^{\beta}, \\
\psi_{\alpha} \phi_{\beta}-\psi_{\beta} \phi_{\alpha} & =\epsilon_{\alpha \beta} \psi^{\gamma} \phi_{\gamma}, \\
\psi^{\alpha} \phi^{\beta}-\psi^{\beta} \phi^{\alpha} & =-\epsilon^{\alpha \beta} \psi^{\gamma} \phi_{\gamma},
\end{aligned}
$$

\footnotetext{
${ }^{7}$ However the entire responsibility for the publication is ours. The financial support received from TÜBİTAK does not mean that the content of the publication is approved in a scientific sense by TÜBİTAK.
} 


$$
\begin{aligned}
\gamma_{a} \gamma_{b} & =\eta_{a b}+\epsilon_{a b c} \gamma^{c} \\
\left(\gamma^{a}\right)_{\alpha \beta}\left(\gamma_{a}\right)_{\gamma \delta} & =2 \epsilon_{\alpha(\gamma} \epsilon_{\delta) \beta}, \\
V_{\alpha \beta} & =\left(\gamma^{a}\right)_{\alpha \beta} V_{a}=V_{\beta \alpha}, \\
V_{a} & =-\frac{1}{2}\left(\gamma_{a}\right)^{\alpha \beta} V_{\alpha \beta} .
\end{aligned}
$$

Note that a vector index is equivalent to a symmetric pair of spinor indices through the last relation.

$$
\eta_{a b}=\eta_{(\alpha \beta)(\gamma \delta)}=-\epsilon_{\alpha(\gamma} \epsilon_{\delta) \beta} .
$$

Open Access. This article is distributed under the terms of the Creative Commons Attribution License (CC-BY 4.0), which permits any use, distribution and reproduction in any medium, provided the original author(s) and source are credited.

\section{References}

[1] V. Frolov, P. Krtous and D. Kubiznak, Black holes, hidden symmetries, and complete integrability, Living Rev. Rel. 20 (2017) 6 [arXiv:1705.05482] [INSPIRE].

[2] Y. Chervonyi and O. Lunin, Killing(-Yano) Tensors in String Theory, JHEP 09 (2015) 182 [arXiv: 1505.06154] [INSPIRE].

[3] O.P. Santillan, Hidden symmetries and supergravity solutions, J. Math. Phys. 53 (2012) 043509 [arXiv: 1108.0149] [INSPIRE].

[4] G.W. Gibbons, R.H. Rietdijk and J.W. van Holten, SUSY in the sky, Nucl. Phys. B 404 (1993) 42 [hep-th/9303112] [INSPIRE].

[5] F. De Jonghe, K. Peeters and K. Sfetsos, Killing-Yano supersymmetry in string theory, Class. Quant. Grav. 14 (1997) 35 [hep-th/9607203] [inSPIRE].

[6] B. Carter, Global structure of the Kerr family of gravitational fields, Phys. Rev. 174 (1968) 1559 [INSPIRE].

[7] M. Walker and R. Penrose, On quadratic first integrals of the geodesic equations for type [22] spacetimes, Commun. Math. Phys. 18 (1970) 265 [INSPIRE].

[8] G. Papadopoulos, Killing-Yano equations and G-structures, Class. Quant. Grav. 25 (2008) 105016 [arXiv: 0712.0542] [INSPIRE].

[9] G. Papadopoulos, Killing-Yano Equations with Torsion, Worldline Actions and G-Structures, Class. Quant. Grav. 29 (2012) 115008 [arXiv:1111.6744] [INSPIRE].

[10] O. Lunin and J. Tian, Separation of variables in the WZW models, JHEP 06 (2021) 114 [arXiv: 2012.15083] [INSPIRE].

[11] M. Cariglia, G.W. Gibbons, J.W. van Holten, P.A. Horvathy, P. Kosiński and P.M. Zhang, Killing tensors and canonical geometry, Class. Quant. Grav. 31 (2014) 125001 [arXiv: 1401.8195] [INSPIRE].

[12] M. Cariglia, Hidden Symmetries of the Dirac Equation in Curved Space-Time, Springer Proc. Phys. 157 (2014) 25 [arXiv:1209.6406] [INSPIRE]. 
[13] P.S. Howe and U. Lindström, Super-Laplacians and their symmetries, JHEP 05 (2017) 119 [arXiv: 1612.06787] [INSPIRE].

[14] P.S. Howe and U. Lindström, Notes on Super Killing Tensors, JHEP 03 (2016) 078 [arXiv: 1511.04575] [INSPIRE].

[15] P.S. Howe and U. Lindström, Some remarks on (super)-conformal Killing-Yano tensors, JHEP 11 (2018) 049 [arXiv:1808.00583] [InSPIRE].

[16] S.M. Kuzenko, U. Lindström, E.S.N. Raptakis and G. Tartaglino-Mazzucchelli, Symmetries of $\mathcal{N}=(1,0)$ supergravity backgrounds in six dimensions, JHEP 03 (2021) 157 [arXiv: 2012.08159] [INSPIRE].

[17] U. Lindström and M. Roček, Properties of hyperKähler manifolds and their twistor spaces, Commun. Math. Phys. 293 (2010) 257 [arXiv:0807.1366] [INSPIRE].

[18] U. Lindström and Ö. Sarığlu, New currents with Killing-Yano tensors, Class. Quant. Grav. 38 (2021) 195011 [arXiv: 2104.12451] [INSPIRE].

[19] D. Kastor and J. Traschen, Conserved gravitational charges from Yano tensors, JHEP 08 (2004) 045 [hep-th/0406052] [INSPIRE].

[20] D. Kastor, S. Ray and J. Traschen, Do Killing-Yano tensors form a Lie Algebra?, Class. Quant. Grav. 24 (2007) 3759 [arXiv:0705.0535] [INSPIRE].

[21] S. Ölmez, Ö. Sarıŏlu and B. Tekin, Mass and angular momentum of asymptotically AdS or flat solutions in the topologically massive gravity, Class. Quant. Grav. 22 (2005) 4355 [gr-qc/0507003] [INSPIRE].

[22] S. Deser and B. Tekin, Energy in topologically massive gravity, Class. Quant. Grav. 20 (2003) L259 [gr-qc/0307073] [INSPIRE].

[23] J.F. Plebański and M. Demiański, Rotating, charged, and uniformly accelerating mass in general relativity, Annals Phys. 98 (1976) 98 [INSPIRE].

[24] D. Kubiznak and P. Krtous, On conformal Killing-Yano tensors for Plebański-Demiański family of solutions, Phys. Rev. D 76 (2007) 084036 [arXiv: 0707.0409] [INSPIRE].

[25] J.B. Griffiths and J. Podolsky, A new look at the Plebański-Demiański family of solutions, Int. J. Mod. Phys. D 15 (2006) 335 [gr-qc/0511091] [inSPIRE].

[26] J. Podolsky and A. Vratny, New improved form of black holes of type D, Phys. Rev. D 104 (2021) 084078 [arXiv:2108.02239] [INSPIRE].

[27] D. Butter, S.M. Kuzenko, J. Novak and G. Tartaglino-Mazzucchelli, Conformal supergravity in three dimensions: New off-shell formulation, JHEP 09 (2013) 072 [arXiv:1305.3132] [INSPIRE].

[28] S.M. Kuzenko and G. Tartaglino-Mazzucchelli, Conformal supergravities as Chern-Simons theories revisited, JHEP 03 (2013) 113 [arXiv: 1212.6852] [INSPIRE].

[29] S. Deser, R. Jackiw and S. Templeton, Three-Dimensional Massive Gauge Theories, Phys. Rev. Lett. 48 (1982) 975 [inSPIRE].

[30] S. Deser, R. Jackiw and S. Templeton, Topologically Massive Gauge Theories, Annals Phys. 140 (1982) 372 [Erratum ibid. 185 (1988) 406] [INSPIRE].

[31] M. Bañados, C. Teitelboim and J. Zanelli, The black hole in three-dimensional space-time, Phys. Rev. Lett. 69 (1992) 1849 [hep-th/9204099] [INSPIRE]. 
[32] C. Batista, Integrability Conditions for Killing-Yano Tensors and Conformal Killing-Yano Tensors, Phys. Rev. D 91 (2015) 024013 [arXiv:1406.3069] [InSPIRE].

[33] C. Batista, Integrability Conditions for Killing-Yano Tensors and Maximally Symmetric Spaces in the Presence of Torsion, Phys. Rev. D 91 (2015) 084036 [arXiv:1501.05029] [INSPIRE].

[34] T. Houri, D. Kubiznak, C.M. Warnick and Y. Yasui, Local metrics admitting a principal Killing-Yano tensor with torsion, Class. Quant. Grav. 29 (2012) 165001 [arXiv:1203.0393] [INSPIRE]. 